Special issue of the 3rd International Conference on Computational and Experimental Science and Engineering (ICCESEN 2016)

\title{
Photovoltaic Panel Simulation Using a Mobile Application
}

\author{
B. KIRIŞ ${ }^{a, *}$, O. BINGÖL ${ }^{b}$ AND M.H.B. UÇAR ${ }^{c}$
}

${ }^{a}$ Department of Electronic Computer Education, Suleyman Demirel University, 32200 Isparta, Turkey

${ }^{b}$ Faculty of Technology, Electrical and Electronics Engineering, Suleyman Demirel University, 32200 Isparta, Turkey

${ }^{c}$ Faculty of Technology, Department of Information Systems Engineering, Kocaeli University, 41380 Kocaeli, Turkey

Today, the use of renewable energy resources is increasing rapidly because the solar energy is easily accessible and abundant. Solar energy is converted into electrical energy using photovoltaic panels. Photovoltaic panel is an energy source, which contains many solar cells, absorbing the solar energy. Factors affecting the operation of photovoltaic panels are the solar irradiance, the temperature, the surface, and the direction angle. For this reason, before employing a photovoltaic panel, the power values, which can be obtained from such photovoltaic panel should be analyzed and evaluated according to the application conditions, considering all these factors. In this study, calculation of power, current and voltage, generated by a photovoltaic panel, was performed on a mobile device for a determined region. Hourly, daily and monthly total power information, obtained according to the characteristics of the photovoltaic panel, such as panel position, direction and inclination, that can be obtained using the mobile device, are presented to the user by the developed application.

DOI: 10.12693 /APhysPolA.132.922

PACS/topics: 88.40.-j, 88.40.H

\section{Introduction}

The energy needed for technology age is the most important topic. Sun is the natural energy source, which produces the least damage to the nature and is easily available for everyone $[1,2]$.

Solar cells are photovoltaic (PV) devices, converting the Sun energy directly into the electrical energy. The current and voltage, provided by the smallest structure unit of a PV system are very small [2]. To get a usable energy, in a PV panel, a lot of solar cells must be connected in series or in parallel with each other. Panels can be also connected in series or in parallel with each other $[3,4]$.

The electrical energy produced by PV panels can be affected by the radiance, the temperature, the angle of the surface and shading $[5,6]$. By measuring these parameters with the accelerometer, compass and direction sensors available on a mobile devices, one can obtain realistic simulation results. Before making the solar panel application, all of these factors must be considered and the yield, to be obtained from the solar panel, must be analyzed and calculated.

In this study it is aimed to calculate the power generated by a PV panel, using a mobile device. Solar radiation falling onto the area, location, direction and tilt have been obtained using the mobile device itself. Hourly, daily and monthly total power information, predicted for a given PV panel, will be presented to the user, so that mobile device simulation data can be available before the implementation of PV panel.

*corresponding author; e-mail: berkaykiris@gmail.com

\section{Modeling of photovoltaic system}

\subsection{PV cell model}

The equivalent circuit model of the PV cell is shown in Fig. 1. The circuit includes a current source, a diode, a parallel resistor and a series resistor. Current source $I_{\mathrm{ph}}$, represents the current generated by photons. The value of this current is constant at fixed radiation and temperature. The parallel resistance $R_{\mathrm{sh}}$ represents the leakage current and the series resistance $R_{\mathrm{s}}$, represents the voltage value at the output [7]. The shunt resistance $R_{\text {sh }}$ is usually much higher than the load resistance, whereas the series resistance $R_{\mathrm{S}}$ is much smaller than the load resistance [8].

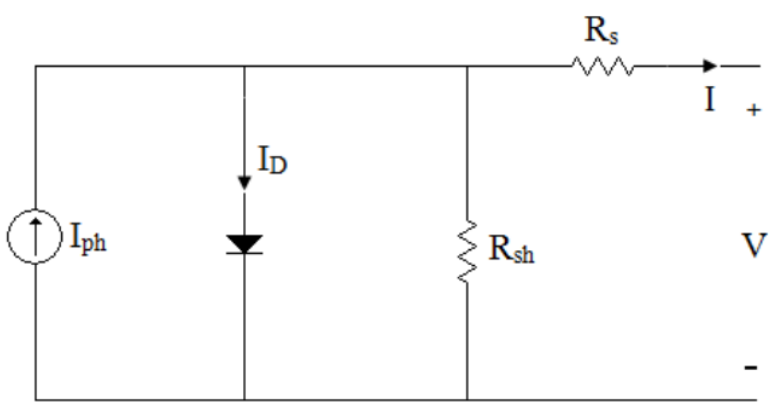

Fig. 1. Equivalent circuit model of a PV cell.

The $I-V$ (current-voltage) characteristic equation of a PV cell is shown in Eq. 1.

$$
I=I_{\mathrm{ph}}-I_{0}\left(\mathrm{e}^{\frac{q\left(V+I R_{S}\right)}{k T A}}-1\right)-\frac{V+I R_{\mathrm{s}}}{R_{\mathrm{sh}}} .
$$

Mathematical translation equations are given as follows,

$$
I_{\text {new }}=I_{\text {ref }}+\left[\alpha\left(\frac{G}{G_{\text {ref }}}\right)\left(T_{\mathrm{c}}-T_{\text {cref }}\right)\right.
$$




$$
\begin{aligned}
& \left.+\left(\frac{G}{G_{\text {ref }}}-1\right) I_{\mathrm{sc}}\right] \\
& \Delta I=\left[\alpha\left(\frac{G}{G_{\text {ref }}}\right)\left(T_{\mathrm{c}}-T_{\text {cref }}\right)+\left(\frac{G}{G_{\text {ref }}}-1\right) I_{\mathrm{sc}}\right] \\
& V_{\text {new }}=-\beta\left(T_{\mathrm{c}}-T_{\text {cref }}\right)-R_{\mathrm{s}} \Delta I+V_{\text {ref. }}
\end{aligned}
$$

where $I_{\text {new }}$ and $V_{\text {new }}$ are the measured current and voltage respectively, $G_{\text {ref }}$ and $G$ are the reference and the measured irradiances, $\alpha$ is the temperature coefficient of the short circuit current, $T_{\text {cref }}$ and $T_{\mathrm{c}}$ are the reference and the measured temperatures, $\beta$ is the temperature coefficient of the module voltage, $T$ is the cell temperature, $V_{T}$ is the thermal voltage $V_{T}=\frac{k T}{q}, R_{\mathrm{s}}$ is series resistance and $R_{\mathrm{sh}}$ is the shunt resistance [9].

\section{2. $P V$ panel parameters}

The operation of a PV cell is directly affected by the amount of solar radiation and temperature. The output power of a PV cell varies proportionally with solar radiation, but is inversely proportional to temperature. Solar radiation as function of locations and date is calculated in [10]. The performance of a solar collector is highly influenced by its orientation and its angle of tilt with respect to the horizontal. The orientation and tilt angle of a solar collector have strong influence on its performance [11].

The angle of incidence of the direct solar radiation on the tilted surface, $\theta$, and the solar declination, $\delta$, were estimated by the following equations:

$$
\begin{aligned}
& \cos \theta=(A-B) \sin \delta \\
& \quad+[C \sin w+(D+E) \cos w] \cos \delta, \\
& A=\sin \varphi \cos \beta, B=\cos \varphi \sin \beta \cos \gamma, C=\sin \beta \sin \gamma, \\
& D=\cos \varphi \cos \beta, E=\sin \varphi \sin \beta \cos \gamma,
\end{aligned}
$$

where $\varphi$ is the latitude of site, $\gamma$ is the surface azimuth angle, $w$ is the hour angle.

If the angle of the radiation with respect to the surface normal is $\theta$, the following equation is obtained for the output current,

$$
I_{\theta}=I_{\text {new }} \cos \theta \text {. }
$$

\section{Overview of hybrid mobile application}

There are multiple ways to develop a mobile application. Frameworks for cross-platform development can be divided into four classes: the web approach, the hybrid approach, the interpreted approach and the crosscompiled approach [12]. The hybrid approach (HA) relies on a WebKit rendering engine to show on the smartphone a web application [12]. Hybrid mobile apps are developed using both web and mobile technologies. A Hybrid app is partly a native app, and partly a web app [13]. An example of this class of frameworks is PhoneGap, also known as Apache Cordova [12].

PhoneGap is an open source development framework for creating cross-platform mobile apps using web technologies, incorporated into the Apache incubator, named as "Apache Cordova". PhoneGap's approach is to use the device's web browser as an intermediate level of abstraction, that permits to implement the logic layer based on JavaScript, and the presentation layer based on HTML and CSS. PhoneGap architecture is shown in Fig. 2. PhoneGap supports all major mobile operating systems (e.g. Android, iOS, RIM, Windows Mobile, etc.), although in some of them it does not provide full management of functionality of the device [14]. The Cordova library is available, which provides hardware and sensor information of the mobile device. It provides customized JavaScript methods and verifiers, especially for GPS, contacts, camera, accelerometer, connection, devices, files, events, geolocation and storage [15]. PhoneGap apps, while written in Javascript, are installed like native apps. The advantage is that no several code versions are required for different mobile platforms [16].

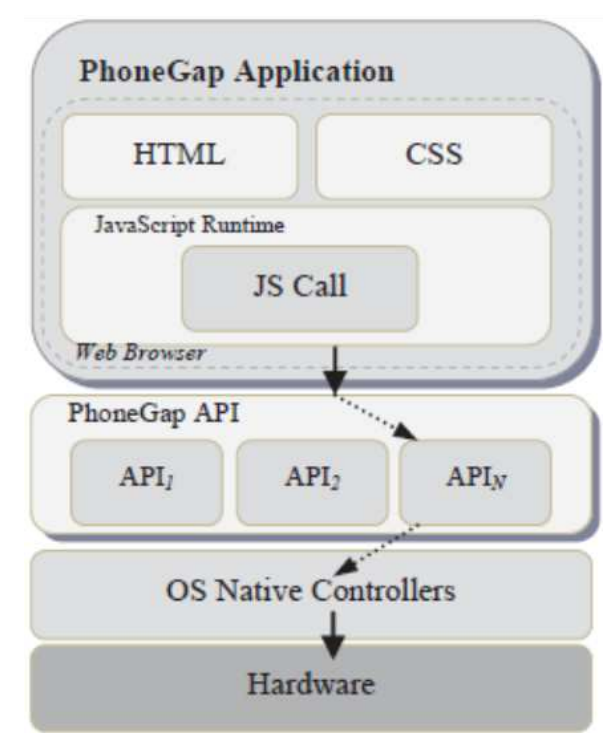

Fig. 2. PhoneGap application architecture.

\section{System design and implementation}

The block diagram of the system is shown in Fig. 3.

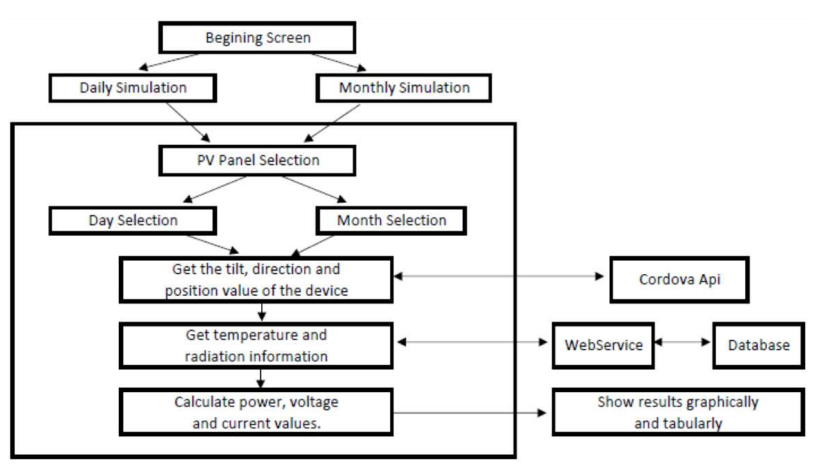

Fig. 3. Block diagram of the system. 


\subsection{Software design}

The simulation process was performed in the PhoneGap development environment, MSSQL database and $\mathrm{C} \#$ web service. By means of the accelerometer, compass, direction sensors, found on the mobile device it is possible to obtain the parameters that determine the power of a PV panel.

Using PhoneGap plugins, the tilt of the device is obtained. The direction information of the device is obtained by device orientation and location information is obtained by geolocation. The features of the PV panels available in the catalogs (datasheets), offered by the manufacturers, have been added to the system. Temperature and solar radiation information cannot be obtained correctly using the device. For this, the data obtained from the SODA (Solar Energy Services for Professionals) service can be stored in the database and thus the radiation and temperature information for any date can be easily obtained [17].

Figure 4 shows the display of the program's launch screen on the mobile device and the factors affecting the PV panel efficiency.

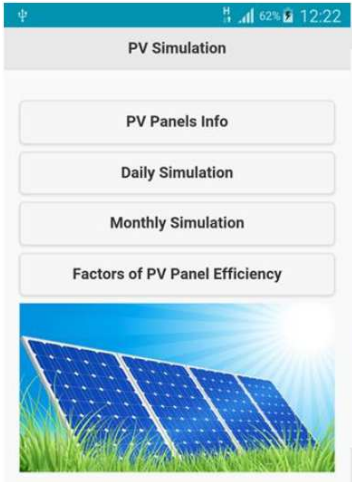

(a)

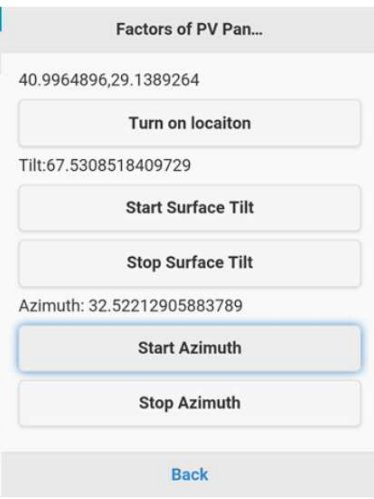

(b)
Fig. 4. (a) Start form, (b) factors of PV panel efficiency form.

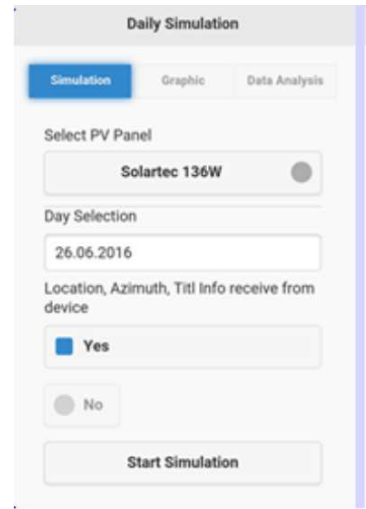

(a)

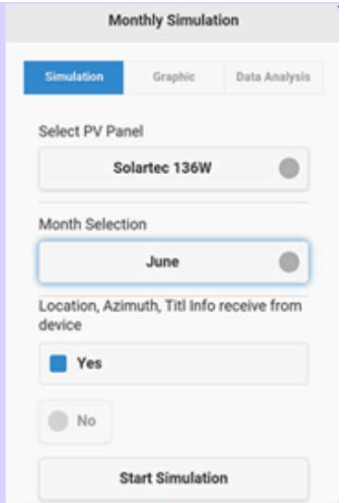

(b)
Fig. 5. (a) Daily simulation start form, (b) monthly simulation start form.
Simulation process can be performed separately by selection of daily or monthly simulation. Daily and monthly simulation screens are shown in Fig. 5. After selecting the PV panel and selecting the date, current, voltage and power values are calculated based on the parameters obtained from the mobile device.

The flow chart diagram of the operation of the system is shown in Fig. 6.

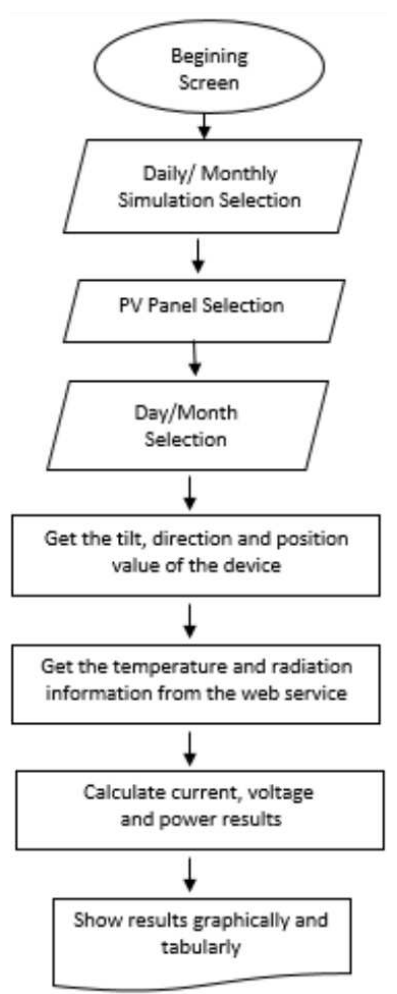

Fig. 6. Flowchart of the system.

\subsection{Simulation application}

For the daily simulation application, Kocaeli, on date of 10/06/2016 and Solartec 136W PV panel were selected. In the first simulation, $16^{\circ}$ surface tilt angle and $97^{\circ}$ azimuth angle of the mobile device were measured on the panel surface and the calculation was performed according to these values. The monthly simulation is started for June, with the same values as the daily simulation.

The same day and same PV panel selection was made for the second day simulation. Recalculation was performed by measuring $20^{\circ}$ surface tilt angle and $300^{\circ}$ azimuth angle. The monthly simulation has been restarted in June with these values. Table I shows the comparison of the daily and monthly simulation results.

In the daily simulation test the first simulation predicted $700.4 \mathrm{~W}$ of power and the next simulation predicted $719.2 \mathrm{~W}$ of power. The second day simulation is by $2.6 \%$ more successful than the simulation of the previous day. 
TABLE I

Simulation results.

\begin{tabular}{c|c|c|c|c}
\hline \hline Simulation type & Daily & Daily & Monthly & Monthly \\
\hline PV panel & \multicolumn{4}{|c}{ Solartec 136W } \\
\hline Simulation date & $10 / 06 / 2016$ & \multicolumn{2}{c}{ June } \\
\hline Surface tilt [Degrees] & 16 & 20 & 16 & 20 \\
Azimuth [Degrees] & 97 & 300 & 97 & 300 \\
PV panel power [W] & 700.4 & 719.2 & 18575.8 & 18961.0
\end{tabular}

In the monthly simulation test, the first simulation predicted $18575.8 \mathrm{~W}$ and the second simulation predicted a total power of $18961.0 \mathrm{~W}$. The second-monthly simulation has shown a better result, by $2 \%$, than the previous simulation. The time-power and day-power plots of the second simulation are shown in Fig. 7. It has been observed that different results are obtained with the mobile application, when the surface gradient and azimuth angle change.

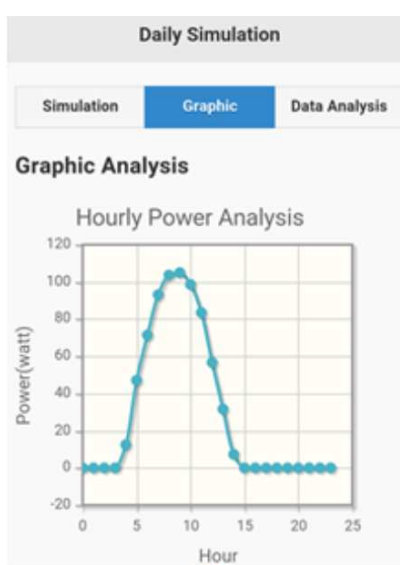

(a)

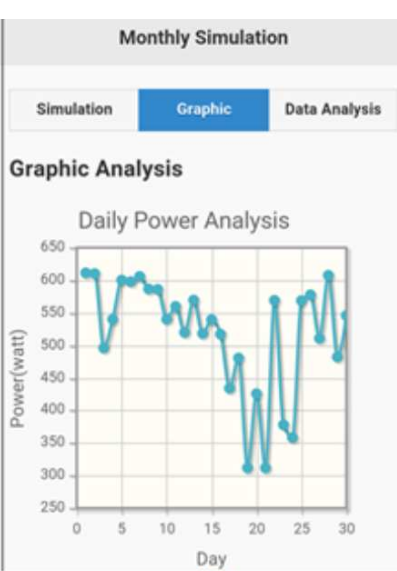

(b)
Fig. 7. (a) Daily simulation and (b) monthly simulation graphs.

\section{Conclusions}

In recent years, solar panel applications in our country have been frequently seen in solar panel fields and roof applications. The high cost of PV panels limits their areas of use. The efficiency of PV panels is directly related to the environmental factors and the layout of the panels. For this reason, it is absolutely necessary to analyze the conditions of the zone before implementation of PV panels.
With this study, before the implementation of the PV panel, a mobile application was created to analyze the system in accordance with actual conditions. Today, mobile applications and renewable energy become more important and system analysis is accelerated and simplified.

\section{References}

[1] M. Cetin, N. Egrican, Energy Policy 39, 7184 (2011).

[2] B. Kiriş, O. Bingöl, R. Şenol, A. Altintaş, Acta Phys. Pol. A 130, 55 (2016).

[3] A. Chouder, S. Silvestre, J. Solar Energy Engin. 131, 024504 (2009).

[4] H. Tian, F. Mancilla-David, K. Ellis, E. Muljadi, P. Jenkins, Solar Energy 95, 1 (2013).

[5] K. Ishaque, Z. Salam, Solar Energy 85, 2217 (2011).

[6] R. Ramabadran, B. Mathur, Modern Appl. Sci. 3, 1 (2009).

[7] G. Bayrak, M. Cebeci, Erciyes Üniv. Bilimleri Enstitüsü Dergisi 28, 198 (2012).

[8] A.A. Jadallah, D.Y. Mahmood, Z.A. Abdulqader, Acta Phys. Pol. A 128, B-461 (2015).

[9] A. Chouder, S. Silvestre, N. Sadaoui, L. Rahmani, Simulation Modell. Pract. Th. 20, 46 (2012).

[10] Z. Er, Acta Phys. Pol. A 130, 72 (2016).

[11] H. Gunerhan, A. Hepbasli, Build. Environment 42, 779 (2007).

[12] M. Ciman, O. Gaggia, Pervasive Mobile Comput. 39, 214 (2016).

[13] C. Freirea, M. Painhoa, Procedia Technol. 16, 481 (2014).

[14] L. Corral, A. Sillitti, G. Succi, Procedia Comput. Sci. 10, 736 (2012).

[15] B. Kiriş, M.Sc. Thesis, Kocaeli Üniversity, 2016.

[16] K. Sankaran, A. Akkihebbal, M.C. Chana, L. Pehb, Computer Communicat. 73, 56 (2016).

[17] Solar Energy Services for Professionals, 2014. 